\title{
DYNAMIC WETTING AND DEWETTING: COMPARISON OF EXPERIMENT WITH THEORIES
}

\author{
Evgeniya.G. Orlova ${ }^{1,{ }^{*}, \text { Ekaterina R. Malyhina }}{ }^{1}$, Dmitriy.V. Feoktistov ${ }^{1}$, and Larisa.S. \\ Zhidkova $^{1}$ \\ ${ }^{1}$ National Research Tomsk Polytechnic University, 634050 Tomsk, Russia
}

\begin{abstract}
The dynamics wetting/dewetting of a metal surface by distilled water drop was studied experimentally. The advancing and receding dynamic contact angles were obtained as a function of a contact line speed. The hydrodynamic and molecular-kinetic models have been applied to the experimental data to interpret the obtained results. The independent variables of the molecular-kinetic and hydrodynamic models, and the determination coefficient were determined by fitting procedure. The receding contact angles are found to be fitted better to the wetting models in comparison with the advancing dynamic contact angles.
\end{abstract}

\section{Introduction}

Technological processes of spray cooling, cultivated crop dusting, spin soldering, spray coating, DNA synthesis are based on the drop spreading and evaporation phenomena [1-7].

When the liquid is moving over the solid substrate, a dynamic contact angle (DCA) is established. This angle reflects the balance between driving and drag forces and is a function of a three-phase contact line (CL) speed.

To describe the physical process of spreading, several theories predicting the relationship between the DCA and the CL speed were developed. These theories can be classified according to the mechanisms of energy dissipation in the vicinity of the contact line: molecular-kinetic (MKT) $[8,9]$ and hydrodynamic $[10,11]$ theories.

The molecular kinetic theory considers the molecular jumps at the CL as a rateactivated, with an equilibrium frequency $k_{o}$ and a displacement distance $\lambda$. So, the relationship between the DCA $\theta_{D}$ and the CL speed $U$ is described by [8]:

$$
\theta_{D}=\arccos \left[\cos \theta_{S}-\left(\frac{2 k_{B} T}{\sigma_{L V} \lambda^{2}}\right) \arcsin \left(\frac{U}{2 k_{0} \lambda}\right)\right]
$$

where $k_{B}$ is the Boltzmann constant, $T$ the liquid temperature, $\theta_{S}$ the static contact angle, and $\lambda$ and $k_{0}$ are parameters corresponding to the length of displacement and frequency constant for molecular displacements of the contact line.

\footnotetext{
* Corresponding author: lafleur@tpu.ru
} 
Nowadays, there is no definitive way of predicting the constants $\lambda$ and $k_{0}$ apriori for a given solid/liquid system. For this reason, these parameters are usially obtained from experiment by curve fitting procedures [12]. The experimentally determined parameters obtaind by different scientific groups are listed in Table 1 .

Table 1. Fitting parameters of molecular-kinetic model obtained in some works.

\begin{tabular}{|c|c|c|c|c|}
\hline Reference & Liquid & $\theta_{S},{ }^{\circ}$ & $\lambda, \mathrm{nm}$ & $k^{0}, \mathrm{~Hz}$ \\
\hline$[13]$ & water & - & 0.3 & $2 \cdot 10^{10}$ \\
\hline$[14]$ & water & 82 & 0.36 & $8.6 \cdot 10^{9}$ \\
\hline$[15]$ & water & 54.1 & 1.02 & $1.19 \cdot 10^{3}$ \\
\hline$[16]$ & water & 55.5 & 1.79 & $6.67 \cdot 10^{5}$ \\
\hline$[17]$ & glycerine solution & - & 7.3 & $3.4 \cdot 10^{7}$ \\
\hline
\end{tabular}

According to the hydrodynamic model the moving process of the contact line is dominated by the viscous dissipation of the liquid; and the bulk viscous friction is the main resistance force for the contact line motion. The shear flow in the vicinity of the contact line leads to a viscous bending of the liquid/vapor interface. For this reason, the dynamic contact angle deviates from its equilibrium value, and can be defined as [10]:

$$
\theta_{D}^{3}=\theta_{S}^{3} \pm\left(\frac{9 \mu U}{\sigma_{L V}}\right) \ln \left(\frac{L}{L_{m}}\right)
$$

where $L$ is a characteristic capillary length, and $L_{m}$ the slip length. In Eq. (2) the plus sign applies to the advancing liquid movement and the minus sign applies to the receding liquid movement.

The capillary length is given by [18]:

$$
L=\sqrt{\frac{2 \sigma_{L V}}{\rho g}}
$$

where $\sigma_{L V}$ is surface tension at the liquid/vapour interface, and $\rho$ the liquid density.

The slip length $L_{m}$ is the fitting parameter. It represents the length of the region where the no-slip boundary condition of classical continuum theory does not hold, so this value should be in the order of molecular dimension [15].

The aim of this work is to obtain the dependences of the advancing and receding dynamic contact angles from the contact line speed of a drop when it spreads over the metal surface. Also the reconciliation of experimental data with the hydrodynamic and molecularkinetic models of wetting dynamics was undertaken.

\section{Materials and methods}

Experimental setup (fig.1) was used to study the wetting and spreading over the solid substrate $[19,20]$.

Experimental setup consists of the electronic syringe pump 1 to supply the distilled water through the opening $2 \mathrm{~mm}$ in diameter from the lower side of the substrate 2 . Thus, the bottom-up method [21] for drop formation $(0.3 \mathrm{ml})$ with a liquid flow rate of $0.04 \mathrm{ml} / \mathrm{s}$ was implemented. The substrate was set on the special platform, which can be heated from the bottom side of the thermoelectric module 3 attached to the aluminium plate of the goniometer 4 . To record the temperature, the chromel-alumel thermocouples 5 were used. 
The light beam from the source 8 passed through a fiber-optic illuminator, and converted to the plane-parallel light. The drop spreading over the surface was lighted, and the shadow images of this process were obtained by the high-speed camera Fastvideo$500 \mathrm{M}$ (with a maximum resolution of $1280 \times 1024$ pixels and at 10 frames per second) equipped with a macro lens.

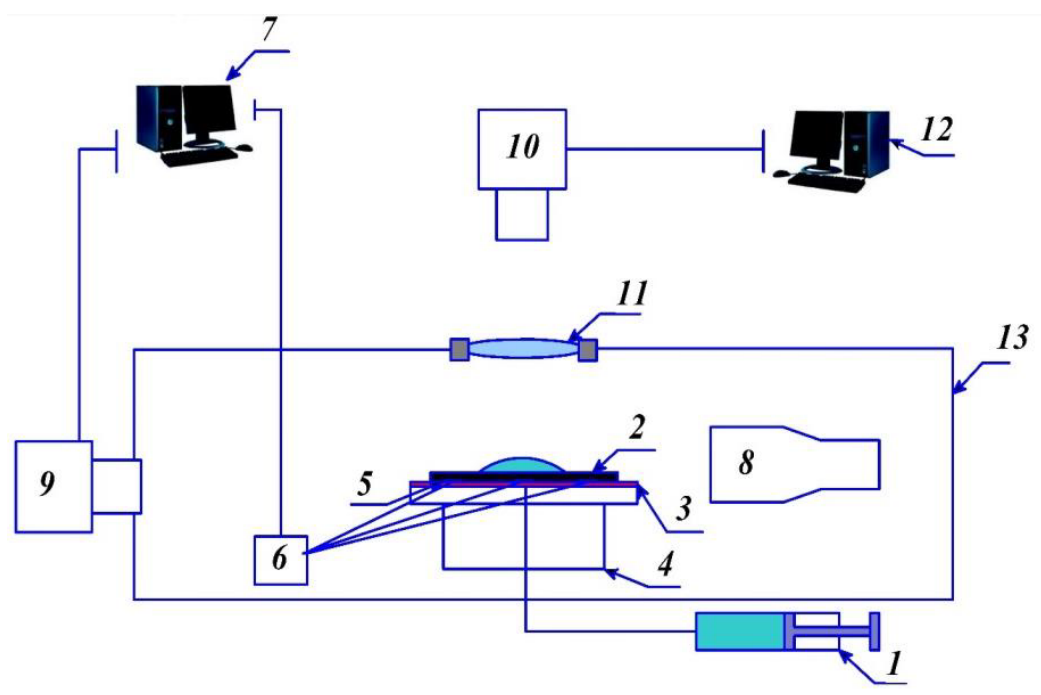

Fig. 1. The scheme of the experimental setup: 1 - syringe pump; 2 - substrate; 3 - thermoelectric module; 4 - goniometer; 5 - thermocouples; 6 - analog-to-digital converter; 7, 12 - personal computers; 8 - light source; 9, 10 - high-speed video cameras; 11 - lens of the upper optical system; 13 - transparent box.

Control of the geometrical shape of the drop was carried out by the high-speed camera 10. To increase the size of the video frames, the lens 11 was used.

The experimental setup is isolated from the external factors changing the temperature, velocity of air flows and environmental humidity by the transparent box 13 made of polymer glass with $3 \mathrm{~mm}$ thick.

The substrate made of brass was used in the experiment. Its surface was studied by profilometer "Micro Measure 3D station". The arithmetic average surface roughness for the brass substrate is equal to $0.016 \mu \mathrm{m}$.

The error of dosing by the syringe pump does not exceed $2 \%$. Random error is calculated from the results of repeated experiments. To do this, from two to six experiments were performed at fixed values of the studied factors. Thereafter, the mean values of the contact angle and standard deviations were defined. The variation coefficients were equal to from $10 \%$ to $14 \%$. Random errors of measurements were mainly due to the small limits when dispensing liquid through a silicone tube.

\section{Results and discussion}

According to results of the experiments the advancing and receding DCA were defined during wetting and dewetting of the substrate. The spreading of the drop starts at contact angle of $83.6^{\circ} \pm 1.5^{\circ}$ that corresponds to the maximal CL speed $(1.76 \mathrm{~mm} / \mathrm{s})$. The spreading ends at the angle of $72.1^{\circ} \pm 1.5^{\circ}$. This value corresponds to the the pre-measured static angle of water/brass/air system $\theta_{s}=70.8 \pm 1.5^{\circ}$. The CL speed in this case is equal to zero. 
Then the drop relaxes [13], and the dewetting starts. The liquid is being withdrawn by the syringe pump, and the receding DCA is being formed.

The hysteresis of the contact angle is defined as the difference between the advancing and receding DCA, when the CL speed is equal to zero (fig. 2).

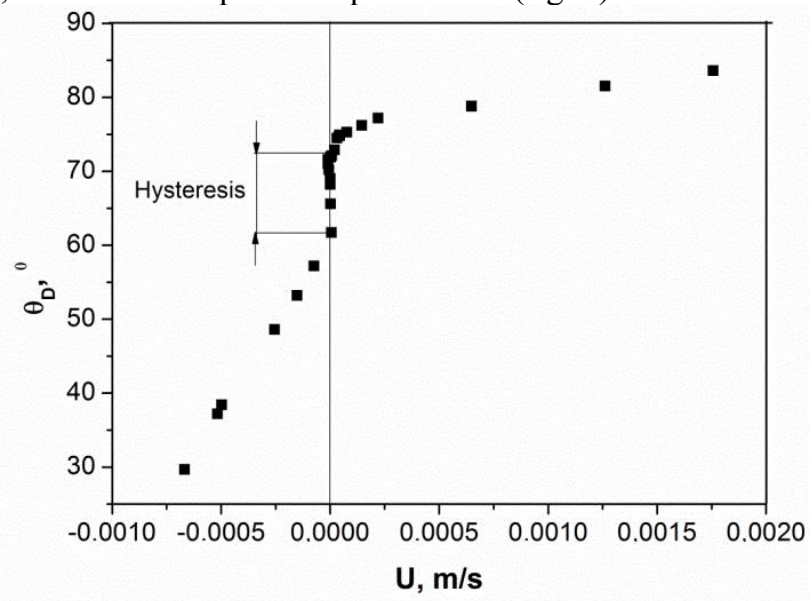

Fig. 2. The advancing and receding dynamic contact angles versus contact line speed. The contact angle hysteresis.

The equations of MKT (1) and hydrodynamic models were used for interpretation of the experimental data and obtaining of functional dependences of the DCA from the CL speed.

Figure 3 shows the dependences of the advancing and receding DCA from the CL speed.

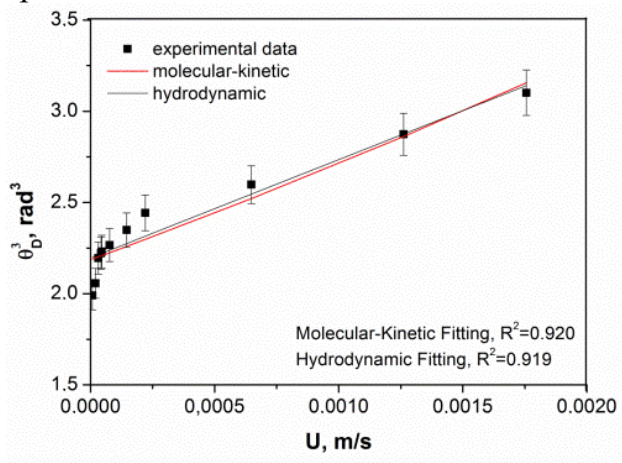

(a)

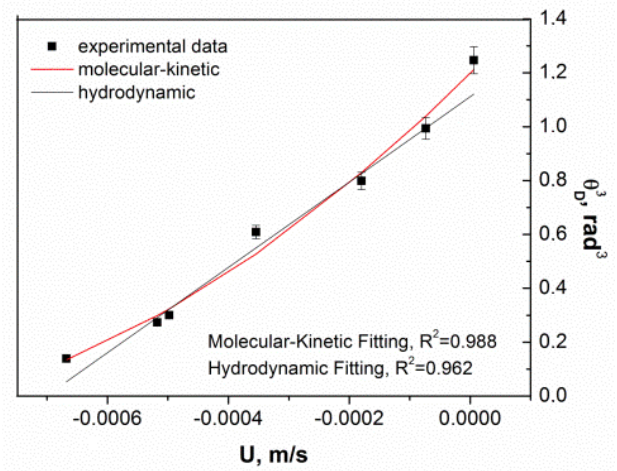

(b)

Fig. 3. Fitting of the MKT and hydrodynamic models to (a) the advancing DCA and (b) the receding DCA.

The treatment of the experimental data was performed by the least squares method. After that, the obtained experimental data of advancing and receding DCA have been separately fitted to the MKT and hydrodynamic wetting models. To estimate the reconciliation of the experiment and models, determination coefficient $\mathrm{R}^{2}$ has been obtained (fig. 3).

According to the results of the reconciliation with the molecular-kinetic model two independent variables were defined: the length of displacement and frequency constant for molecular displacements of the contact line. An initial guess input of $k^{0}$ и $\lambda$ were taken from Table 1. 
The value of the $\ln \left(\frac{L}{L_{m}}\right)$ parameter was determined at fitting the experimental data with the hydrodynamic model. The value of the $L_{m}$ parameter are not presented separately, because it is not physically meaningful. Probably, this is due to the fact that the value of the capillary number $C a=\frac{\mu U}{\sigma_{L V}}$ in Eq. (2) is smaller, in order of $10^{-6}$ for distilled water, in comparison with the value of $\theta_{S}^{3}$.

The values of the molecular-kinetic and hydrodynamic parameters and $\mathrm{R}^{2}$ values from the fitting for the advancing and receding contact line are listed in Table 2.

Table 2. Fitting parameters of molecular-kinetic and hydrodynamic models obtained in work.

\begin{tabular}{|c|c|c|c|c|c|c|c|c|}
\hline & \multirow{2}{*}{$\begin{array}{c}\theta_{s}{ }^{\circ} \\
\text { (measured) }\end{array}$} & \multicolumn{4}{|c|}{ Molecular-kinetic model } & \multicolumn{3}{|c|}{ Hydrodynamic model } \\
\hline & & $\theta_{s}{ }^{\circ}$ & $k^{0}, 10^{9} \mathrm{~Hz}$ & $\lambda, \mathbf{n m}$ & $\mathbf{R}^{2}$ & $\theta_{s}{ }^{\circ}$ & $\ln \left(\frac{L}{L_{m}}\right)$ & $\mathbf{R}^{2}$ \\
\hline $\begin{array}{c}\text { Advancing } \\
\text { CL }\end{array}$ & \multirow{2}{*}{$70.8 \pm 1.5^{\circ}$} & 74.4 & 8.13 & 0.042 & 0.920 & 62.6 & 44400 & 0.919 \\
\hline $\begin{array}{c}\text { Receding } \\
\text { CL }\end{array}$ & & 60.9 & 6.51 & 0.025 & 0.988 & 58 & 12700 & 0.962 \\
\hline
\end{tabular}

It was found that Eq. (1) and (2) fit well with the experimental data. However the receding contact angles $\theta_{R}=f(U)$ were fitted better in comparison with the advancing DCA $\theta_{A}=f(U)$. It is confirmed by the values of the determination coefficients for the receding contact line $\mathrm{R}^{2}=0.988$ and $\mathrm{R}^{2}=0.962$ for the molecula-kinetic and hydrodynamic models, respectively.

\section{Acknowledgments}

The reported study was supported by the grant of President of Russian Federation for the government support of young Russian scientists (MK-6810.2016.8).

\section{References}

1. W.M. Grissom, F.A. Wierum, Int J Heat Mass Transf. 24, 261 (1981)

2. D.V. Zaitsev, O.A. Kabov, Microgravity Sci Technol. 19, 174 (2007)

3. D.O. Glushkov, G.V. Kuznetsov, P.A. Strizhak, R.S. Volkov, Thermal Science 20, 131 (2016)

4. D.V. Zaitsev, E.A. Chinnov, O.A. Kabov, I.V. Marchuk, Technical Physics Letters 30, 231 (2004)

5. V.P. Lebedev, V.V. Lemanov, S.Ya. Misyura, V.I. Terekhov, Fluid Dynamics 28, 624 (1993)

6. S.Ya. Misyura, International Journal of Heat and Mass Transfer 71, 197 (2014)

7. K. Batischeva, E. Orlova, D. Feoktistov, EPJ Web Conf. 19, 01001 (2014)

8. T. D. Blake, J. M. Haynes, J. Colloid Interface Sci. 30, 421 (1969)

9. E. Ruckenstein, C. S. Dunn, J. Colloid Interface Sci. 59, 135 (1977)

10. R. G. Cox, J. Fluid Mech. 168, 169 (1986)

11. O. V. Voinov, J Fluid Dyn 11, 714 (1976) 
12. T.D. Blake, J Colloid Interf Sci 299, 1 (2006)

13. R. Sedev, Adv Colloid Interface Sci. 222, 661 (2015)

14. T.D. Blake, M. Bracke, Y.D. Shikhmurzaev, Phys. Fluid 11, 1995 (1999)

15. S.R. Ranabothu, C. Karnezis, L.L. Dai, J. Colloid Interf. Sci. 288, 213 (2005)

16. R. Fetzer, J. Ralston, J. Phys. Chem. C. 113, 8888 (2009)

17. J.-H. Kim, H.P. Kavehpour, J.P. Rothstein, Phys. Fluids 27, 032107 (2015)

18. M. Schneemilch, R. A. Hayes, J. G. Petrov, J. Ralston, Langmuir 14, 7047 (1998)

19. E.G. Orlova, G. V. Kuznetsov, D. V. Feoktistov, EPJ Web Conf. 82, 01053 (2015)

20. D. Feoktistov, E. Orlova, A. Islamova, EPJ Web Conf. 110, 01018 (2016)

21. K. Gleason, S.A. Putnam, Langmuir 30, 10548 (2014) 